\title{
Real Time Optimization of Solar Powered Direct Contact Membrane Distillation Based on Multivariable Extremum Seeking
}

\author{
Ayman M. Karam and Taous Meriem Laleg-Kirati
}

\begin{abstract}
This paper presents a real time optimization scheme for a solar powered direct contact membrane distillation (DCMD) water desalination system. The sun and weather conditions vary and are inconsistent throughout the day. Therefore, the solar powered DCMD feed inlet temperature is never constant, which influences the distilled water flux. The problem of DCMD process optimization has not been studied enough. In this work, the response of the process under various feed inlet temperatures is investigated, which demonstrates the need for an optimal controller. To address this issue, we propose a multivariable Newton-based extremum seeking controller which optimizes the inlet feed and permeate mass flow rates as the feed inlet temperature varies. Results are presented and discussed for a realistic temperature profile.
\end{abstract}

\section{INTRODUCTION}

Membrane distillation (MD) is a thermal separation technique driven by a vapor pressure difference across a hydrophobic membrane. In this process, a hot feed stream is passed along one side of a porous hydrophobic membrane. Water vapor is then transferred through the membrane to the other cooler (permeate) side. The vapor then condenses at the membrane-permeate interface and clean water is produced.

MD has emerged as a sustainable water desalination technique, which offers several advantages over conventional desalination methods. In contrast to reverse osmosis, MD operates at a lower water pressure, hence is less susceptible to scaling and membrane fouling. Moreover, MD water desalination can be integrated with renewable and waste energy sources, since the feed water is not heated up to boiling temperature [1]. All of these features reduce the capital and operational cost of the MD process. Over the years, new MD configurations and membrane material were the main area of research [2]. However, very limited work has been done in terms of process control and optimization [3], [4].

As it is well known, the objective is always to operate any process at optimal settings, which reduces the operational costs and guaranties the performance and stability of the system. This is true for the solar-powered MD water desalination, where the objective is to maximize the water production and reduce the energy consumption at the same time through the manipulation of the feed and permeate inlet mass flow rates.

In terms of process optimization, the work in [4] developed a neural network model, which was used to calculate optimal

Ayman Karam and T.M. Laleg-Kirati are with the Computer, Electrical and Mathematical Science and Engineering division (CEMSE) at King Abdullah University of Science and Technology (KAUST), Thuwal, KSA. ayman.karam@kaust.edu.sa, taousmeriem. laleg@kaust.edu.sa feed-forward gains for solar powered MD. However, when coupling the MD with renewable energy, it is important to consider the unsteady nature of the energy source. Therefore, a real time optimal control would be more suitable. The MD water desalination process can be farther explored and a better overall system performance can be achieved.

Several optimal control schemes have been reported in the literature. For instance, model predictive control (MPC) [5], [6] requires full knowledge of the plant model and the objective function. Moreover, MPC for nonlinear system is still challenging and computationally expensive. On the other hand, extremum seeking is another adaptive optimal controller which is finds and tracks the peak of the referenceto-output map in real time without knowledge of either the system model or the objective function. Moreover, ES controller are generally computationally efficient.

In this paper, we present a real time multivariable optimal controller for a solar powered direct contact membrane distillation (DCMD) using the Newton-based extremum seeking (ES) method. This controller takes into account the varying feed inlet temperature, due to the dynamic nature of the sun. Moreover, since ES is non-model based, the controller design is robust against plant-model mismatch. This important since the results obtained in simulation should be applicable to experimental validation.

Stability of a feedback ES controller was proved for a general nonlinear systems in [7]. This scheme approximated the gradient of the input-to-output map and tracked its peak in real time. Based on the so called gradient-based ES, successful applications followed the proof across many fields [8], [9]. For instance, in [9], ES was applied to obtain maximum power point tracking for a photovoltaic (PV) array. However, the convergence of the gradient-based ES schemes depended on an unknown Hessian matrix, which resulted in different convergence speeds for multi-input optimization problems. Therefore, in [10] a Newton-based ES was introduced for a general MIMO system, where an estimate of the inverse of the Hessian is dynamically obtained. This allowed for the convergence rate to be user assigned and independent of the Hessian. The Newton-based ES was experimentally implemented for power optimization of a solar PV array [11], which demonstrated the superiority of the Newton-based ES over the gradient-based ES scheme. Therefore, for this work, the multivariable Newton-based ES was selected for the optimization of the solar powered DCMD water desalination.

The dynamic model of heat and mass transfer in the DCMD process along with the solar powered DCMD optimal control problem formulation is presented in Section 


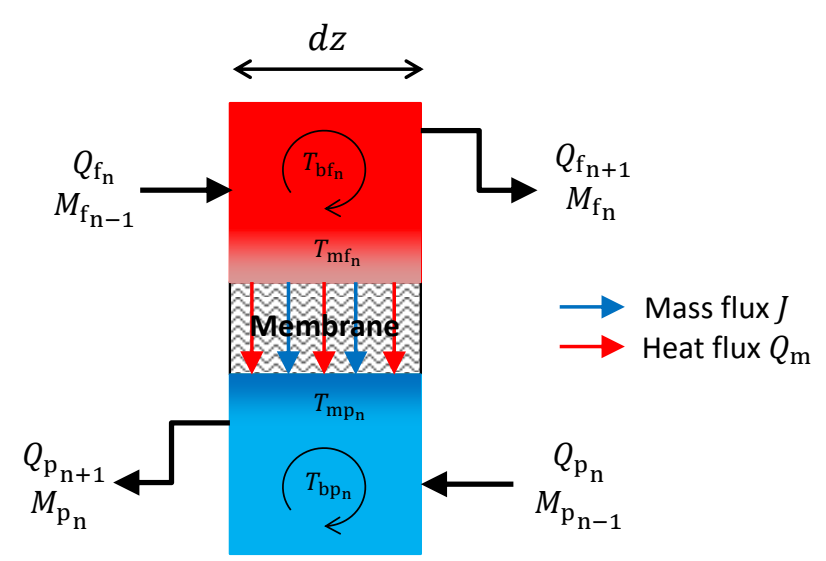

Fig. 1. Schematic diagram of the $\mathrm{n}^{\text {th }}$ DCMD cell.

II. Although ES is non-model based technique, the dynamic model is of DCMD is presented here to demonstrate the method and carry out simulations. Then, the multivariable Newton-based ES method is briefly summarized in Section III. Simulation results are presented and discussed in Section IV. Conclusions are then drawn in Section V.

\section{SOlAR POWEREd DCMD}

We will first focus on the DCMD module. In this configuration, hot water passes along the hydrophobic membrane from one side, called the feed, and cold water flows in the counter direction along the other side, which is called the permeate. Water vapor is driven from the feed side across the membrane and into the permeate side by the induced vapor pressure difference. Both heat and mass transfer processes occur simultaneously as water evaporates at the feed-membrane interface and condenses at the permeatemembrane interface. As a result, the temperature at the membrane boundary layers differs from the bulk temperature of the feed and permeate streams. The DCMD module can be divided into $\mathrm{N}$ control volume cells, each cell has a uniform bulk temperatures $\left(T_{\mathrm{bf}_{\mathrm{n}}}, T_{\mathrm{bp}_{\mathrm{n}}}\right)$ except at the boundary layers where the temperatures are $\left(T_{\mathrm{mf}_{\mathrm{n}}}, T_{\mathrm{mp}_{\mathrm{n}}}\right)$, as shown in Fig.1 for the $\mathrm{n}^{\text {th }}$ cell. A summarized presentation of the mass and heat transfer model is in the following subsections.

\section{A. Mass transfer in DCMD}

The transport phenomena is described by the classic gas permeation and heat transfer theories. The mass flux $(J)$ in DCMD is related to the saturated vapor pressure difference across the membrane $(\Delta P)$ through the membrane mass transfer coefficient $\left(B_{\mathrm{m}}\right)$ as follows [12]

$$
J=B_{m} \Delta P=B_{m}\left(P_{\mathrm{mf}}-P_{\mathrm{mp}}\right) .
$$

The saturated vapor pressure of pure water $\left(P_{w}^{s a t}[T]\right)$ as a function of temperature is given by the Antoine equation [13]:

$$
P_{w}^{s a t}[T]=\exp \left(23.1964-\frac{3816.44}{T+227.02}\right) .
$$

Dissolved salt in the feed stream reduces the saturated vapor pressure. Therefore, to compensate for this the following relation was proposed in [13]:

$$
P_{\mathrm{mf}}=\left(1-x_{N a C l}\right)\left(1-0.5 x_{N a C l}-10 x_{N a C l}^{2}\right) P_{w}^{s a t}\left[T_{\mathrm{mf}}\right],
$$

where $x_{\mathrm{NaCl}}$ is the mole fraction of $\mathrm{NaCl}$ in the feed stream. However, the permeate and the saturated vapor at the membrane-permeate interface is pure water. Thus, $P_{\mathrm{mp}}=$ $P_{w}^{s a t}\left[T_{\mathrm{mp}}\right]$.

\section{B. Heat Transfer in DCMD}

Heat transfer in DCMD is spatially and temporally distributed phenomena. In our previous work, a dynamical model, based on electrical analogy to thermal systems, was proposed and experimentally validated in both transient and steady-state response. The reader is referred to [14], [15] for full details on this model. The resultant model is a system of differential-algebraic equations (DAE) or a descriptor system.

The electrical analog circuit for the DCMD module is shown in Fig. 2. By applying the electrical laws (KCL and $\mathrm{KVL}$ ) on the analogue circuit for DCMD, as seen in Fig. 2, the following equations are obtained:

At the feed side, the rate of change of the heat transfer rate from the $\mathrm{n}-1$ cell to the $\mathrm{n}^{\text {th }}$ cell is proportional to the temperature difference between them. Taking into consideration the series impedance $\mathrm{Z}_{\mathrm{n}}^{\mathrm{f}}$, this is expressed as

$$
\frac{d Q_{\mathrm{f}_{\mathrm{n}}}}{d t}=\frac{1}{\mathrm{~L}_{\mathrm{f}}^{\mathrm{n}}} T_{\mathrm{bf}_{\mathrm{n}-1}}-\frac{\mathrm{R}_{\mathrm{fz}}^{\mathrm{n}}}{\mathrm{L}_{\mathrm{f}}^{\mathrm{n}}} Q_{\mathrm{f}_{\mathrm{n}}}-\frac{1}{\mathrm{~L}_{\mathrm{f}}^{\mathrm{n}}} T_{\mathrm{bf}_{\mathrm{n}}} .
$$

Using Kirchoff's current law at the $\mathrm{n}^{\text {th }}$ feed node, it follows that the rate of change for the bulk feed temperature $\left(T_{\mathrm{bf}_{\mathrm{n}}}\right)$ is

$$
\begin{aligned}
\frac{d T_{\mathrm{bf}_{\mathrm{n}}}}{d t}= & \frac{1}{C_{\mathrm{bf}}} Q_{\mathrm{f}_{\mathrm{n}}}-\frac{1}{C_{\mathrm{bf}}}\left(\frac{1}{\mathrm{R}_{\mathrm{f}}}+J_{\mathrm{n}} A_{m} c_{p}\right) T_{\mathrm{bf}_{\mathrm{n}}} \\
& -\frac{1}{C_{\mathrm{bf}}} Q_{\mathrm{f}_{\mathrm{n}+1}}+\frac{1}{C_{\mathrm{bf}} \mathrm{R}_{\mathrm{f}}} T_{\mathrm{mf}_{\mathrm{n}}} .
\end{aligned}
$$

where (4) describes the dynamics of the heat transfer rates into and out of the $\mathrm{n}^{\text {th }}$ feed cell $\left(Q_{\mathrm{f}_{\mathrm{n}}}\right.$ and $Q_{\mathrm{f}_{\mathrm{n}+1}}$ respectively).

Similarly for the permeate side, the rate of change of the heat transfer rate $\left(Q_{\mathrm{p}_{\mathrm{n}}}\right)$ is

$$
\frac{d Q_{\mathrm{p}_{\mathrm{n}}}}{d t}=\frac{1}{\mathrm{~L}_{\mathrm{p}}^{\mathrm{n}}} T_{\mathrm{bp}_{\mathrm{n}-1}}-\frac{\mathrm{R}_{\mathrm{pz}}^{\mathrm{n}}}{\mathrm{L}_{\mathrm{p}}^{\mathrm{n}}} Q_{\mathrm{p}_{\mathrm{n}}}-\frac{1}{\mathrm{~L}_{\mathrm{p}}^{\mathrm{n}}} T_{\mathrm{bp}_{\mathrm{n}}},
$$

and the dynamics of the bulk permeate temperature $\left(T_{\mathrm{bp}_{\mathrm{n}}}\right)$ is

$$
\begin{aligned}
\frac{d T_{\mathrm{bp}_{\mathrm{n}}}}{d t}= & \frac{1}{C_{\mathrm{bp}}} Q_{\mathrm{p}_{\mathrm{n}}}-\frac{1}{C_{\mathrm{bp}} \mathrm{R}_{\mathrm{p}}} T_{\mathrm{bp}_{\mathrm{n}}}-\frac{1}{C_{\mathrm{bp}}} Q_{\mathrm{p}_{\mathrm{n}}+1} \\
& +\frac{1}{C_{\mathrm{bp}}}\left(\frac{1}{\mathrm{R}_{\mathrm{p}}}+J_{\mathrm{n}} A_{m} c_{p}\right) T_{\mathrm{mp}_{\mathrm{n}}} .
\end{aligned}
$$




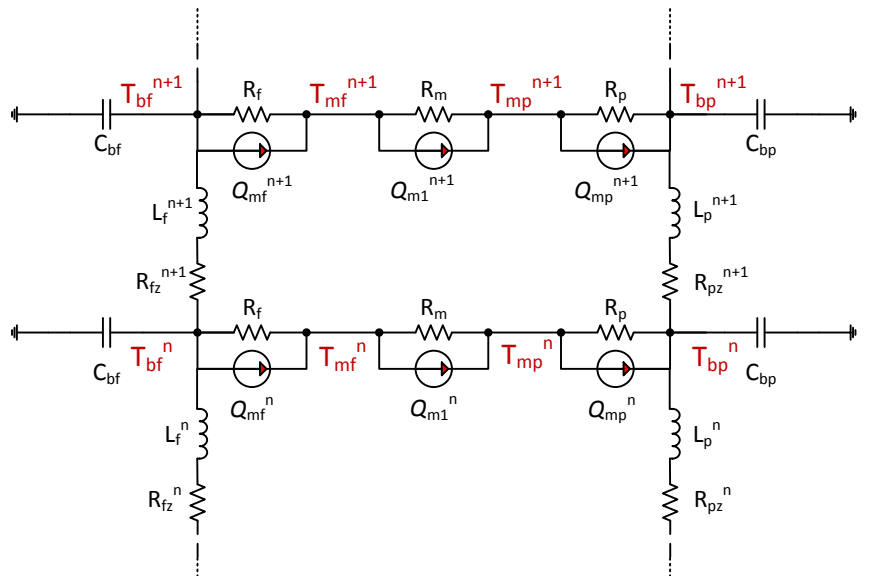

Fig. 2. Completed electrical analogue of the DCMD module.

The coupling between the feed and the permeate dynamics in the $\mathrm{n}^{\text {th }}$ cell is written in residue form as

$$
\begin{aligned}
0 & =\left(\frac{1}{\mathrm{R}_{\mathrm{f}}}+J_{\mathrm{n}} A_{m} c_{p}\right) T_{\mathrm{bf}_{\mathrm{n}}}-\frac{1}{\mathrm{R}_{\mathrm{f}}} T_{\mathrm{mf}_{\mathrm{n}}} \\
& -\left(\frac{1}{\mathrm{R}_{\mathrm{p}}}+J_{\mathrm{n}} A_{m} c_{p}\right) T_{\mathrm{mp}_{\mathrm{n}}}+\frac{1}{\mathrm{R}_{\mathrm{p}}} T_{\mathrm{bp}_{\mathrm{n}}}, \\
0 & =\left(\frac{1}{\mathrm{R}_{\mathrm{m}}}+\frac{1}{\mathrm{R}_{\mathrm{p}}}+J_{\mathrm{n}} A_{m} c_{p}\right) T_{\mathrm{mp}_{\mathrm{n}}}-\frac{1}{\mathrm{R}_{\mathrm{p}}} T_{\mathrm{bp}_{\mathrm{n}}} \\
& -J_{\mathrm{n}} A_{m} H_{v}\left[T_{\mathrm{mf}_{\mathrm{n}}}\right]-\frac{1}{\mathrm{R}_{\mathrm{m}}} T_{\mathrm{mf}_{\mathrm{n}}} .
\end{aligned}
$$

The outlet temperatures at the terminal cells of the feed and permeate analogue are also given by the algebraic equations, which are respectively

$$
\begin{aligned}
& 0=T_{\mathrm{f}_{\text {out }}}-T_{\mathrm{p}_{\text {in }}}-R_{\mathrm{f}_{\text {term }}} Q_{\mathrm{f}_{\mathrm{n}+1}}, \\
& 0=T_{\mathrm{p}_{\text {out }}}-T_{\mathrm{f}_{\mathrm{in}}}+R_{\mathrm{p}_{\text {term }}} Q_{\mathrm{p}_{1}} .
\end{aligned}
$$

The heat and mass transfer equations (4)-(11) represent a nonlinear differential-algebraic system. When considering $\mathrm{N}$ number of interconnected cells, the resultant equations can be expressed as a nonlinear descriptor system of the form

$$
\begin{aligned}
E \dot{X}(t) & =F(X(t), u(t)) \\
J & =g(X)
\end{aligned}
$$

where $X \in \mathbb{R}^{6 \mathrm{~N}+4}$ represents the differential and algebraic states, $\dot{X}$ refers to the time derivative of the state vector, $E \in \mathbb{R}^{6 \mathrm{~N}+4 \times 6 \mathrm{~N}+4}$ is a singular matrix, $F(X(t), u(t))$ is a nonlinear function of the states and input, $u(t)=$ $\left[M_{\mathrm{f}_{\mathrm{in}}}, M_{\mathrm{p}_{\mathrm{in}}}\right]$ represents the inlet feed and permeate mass flow rates respectively, the distilled water flux $J$ is also given by a nonlinear function of the state $g(X)$.

Thus far, the dynamical model of DCMD has been presented. We can now consider the complete setup of the solar powered DCMD water desalination system. This will be discussed in the next subsection.

\section{Optimal control problem of solar powered DCMD}

The solar powered DCMD water desalination setup is composed of a solar thermal collector connected a storage tank and a heat exchanger. The feed water is circulated from the feed tank through the heat exchanger to DCMD module and back to the feed tank. The permeate is pumped from a fresh water tank into the DCMD module, where it collects the distilled water flux and flow back into the fresh water tank. The setup is depicted in Fig. 3, where the control inputs are the mass flow rates of feed and permeate sides. It is clear that the feed inlet temperature $\left(T_{\mathrm{f}_{\mathrm{in}}}\right)$ will vary according to the solar radiation throughout the day, which affects the distilled water flux. Optimal control strategy is needed to ensure the efficiency of the process under disturbed operating conditions. One way to optimize the process is by maximizing the following objective function $\mathcal{Y}$, which minimizes the feed/permeate inlet mass flow rates and maximizes the distilled water flux $(J)$ :

$$
\max \mathcal{Y}=\max \left(\alpha_{1} J-\left(\alpha_{2} M_{\mathrm{f}_{\mathrm{in}}}+\alpha_{3} M_{\mathrm{p}_{\mathrm{in}}}\right)\right)
$$

Fig. 4 shows the response of the DCMD process under various feed inlet temperatures and feed inlet mass flow rates. In this simulation, the permeate inlet mass flow rate was kept constant at $256 \mathrm{Kg} / \mathrm{hr}$, while the feed inlet mass flow rate was increased from $90 \mathrm{Kg} / \mathrm{hr}$ to $375 \mathrm{Kg} / \mathrm{hr}$. This was repeated for 5 feed inlet temperatures. As demonstrated in the figure, increasing the feed inlet temperature increased the distilled water flux. While increasing the feed inlet mass flow rate increases the flux, the flux reaches saturation values at high feed inlet mass flow rates. This behavior provokes the following question: What is the optimal feed/permeate flow rate for a given feed inlet temperature?

It is shown in Fig. 5 that equation (14) has a maximum that varies for different feed inlet temperature. Similar characteristics for the objective function with respect to the inlet permeate mass flow rate. Therefore, the controller is required to automatically track the peak of the objective function by manipulating the inlet feed and permeate mass flow rates. In the next section, the basic concept of the multivariable extremum seeking will be briefly presented.

\section{Multivaraible Extremum Seeking for Real TIME OPTIMIZATION}

Extremum seeking is a real time optimization method, which is particularly useful to keep the reference-to-output map at its extremum (maximum or minimum). This extremum might shift with the process parameters or under disturbance influence and it's the job of ES controller to track the extremum online as it shifts. Newton-based multivariable ES relies on sinusoidal perturbation signals to estimate the gradient and the Hessian of the objective function by using low and high pass filters.

As it has been shown in the previous section, the objective function has a $\left(T_{\mathrm{f}_{\mathrm{in}}}\right)$ dependent peak. Therefore, the following can be assumed: 


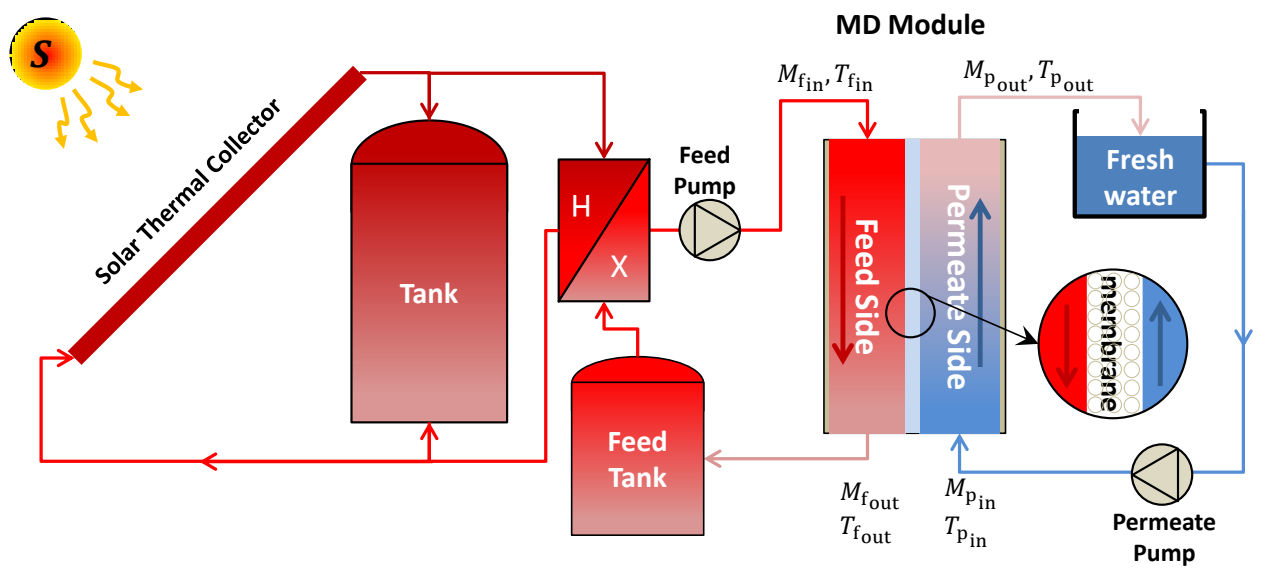

Fig. 3. Schematic diagram of solar powered DCMD setup.

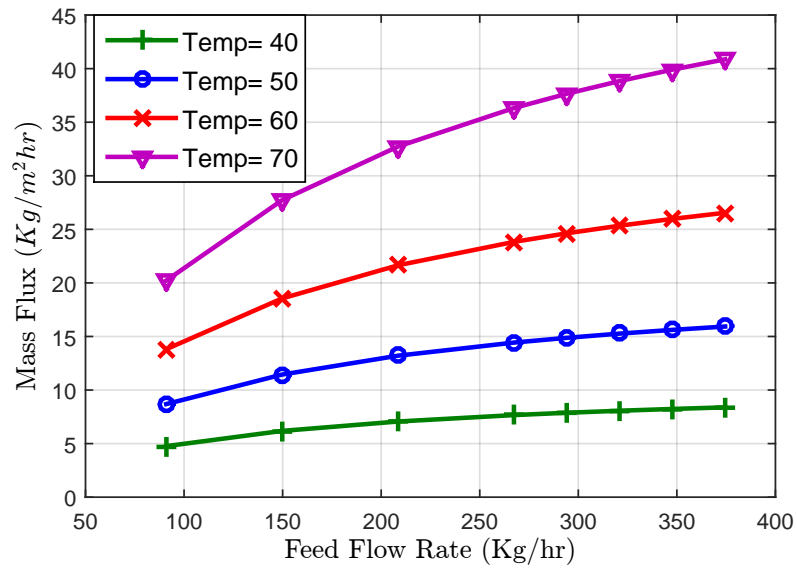

Fig. 4. The vapor flux response as a function of the feed inlet mass flow rate for various feed inlet temperatures.

Assumption 1: There exists $u^{*}=\left[M_{\mathrm{f}_{\mathrm{in}}}^{*}, M_{\mathrm{p}_{\mathrm{in}}}^{*}\right]^{T}$ such that

$$
\begin{aligned}
\nabla \mathcal{Y}\left(u^{*}\right) & =0 \\
\frac{\partial^{2} \mathcal{Y}\left(u^{*}\right)}{\partial u^{2}} & =H \prec 0, \quad H=H^{T} .
\end{aligned}
$$

Suppose that the control input is given by $u=\beta(X, \theta)$, which is a smooth function of the states and the parameters $\theta=\left[\theta_{1}, \theta_{2}\right]^{T}$, where $\theta_{1}, \theta_{2}$ are the inlet mass flow rates for the feed and permeate sides respectively. Then, the closed loop system is given by $E \dot{X}=F(X, \beta(X, \theta))$. The objective of the ES controller is to maximize the value of $\mathcal{Y}$. The block diagram of the Newton-based ES is shown in Fig.6, where $K_{n}$ is a positive diagonal matrix, and the perturbation matrices $\left(L(t) \in \mathbb{R}^{2 \times 1}, \Omega(t) \in \mathbb{R}^{2 \times 2}\right.$, and $\left.\varphi(t) \in \mathbb{R}^{2 \times 1}\right)$ are defined as

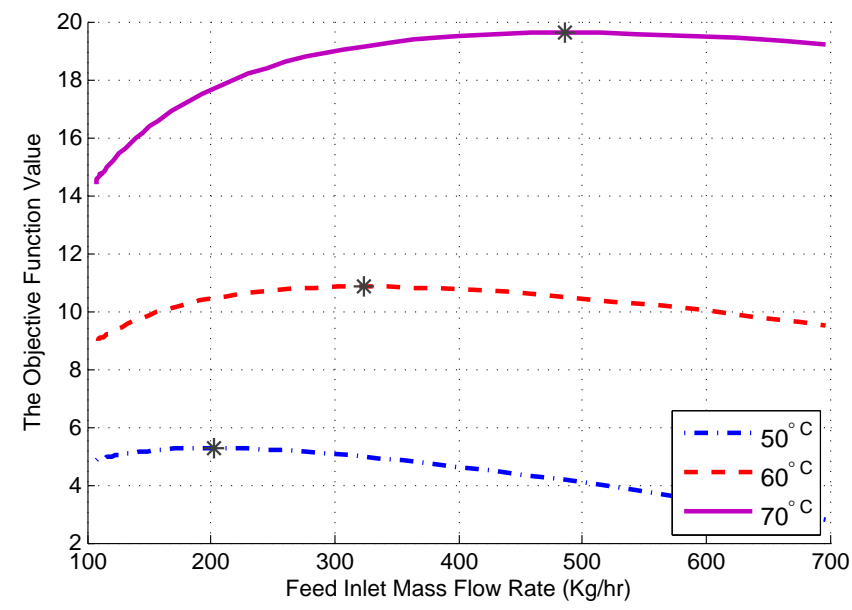

Fig. 5. The objective function at three feed inlet temperatures.

$$
\begin{aligned}
L(t) & =\left[\frac{2}{c_{1}} \sin \left(\omega_{1} t\right), \frac{2}{c_{2}} \sin \left(\omega_{2} t\right)\right]^{T}, \\
\Omega(t) & =\Omega^{T}(t), \\
\Omega_{i, i} & =\frac{16}{c_{i}^{2}}\left(\sin ^{2}\left(\omega_{i} t\right)-\frac{1}{2}\right), \\
\Omega_{i, k} & =\frac{4}{c_{i} c_{k}}\left(\sin \left(\omega_{i} t\right) \sin \left(\omega_{k} t\right)\right), \quad i \neq k, \\
\varphi(t) & =\left[c_{1} \sin \left(\omega_{1} t\right), c_{2} \sin \left(\omega_{2} t\right)\right]^{T},
\end{aligned}
$$

where $\omega_{i} \neq \omega_{k}$ and such that $\omega_{i} / \omega_{k}$ is a rational number, and $c_{1}, c_{2}$ are real positive numbers. The cut-off frequencies for the low and high pass filters , $\omega_{l}$ and $\omega_{h}$ respectively, are designed appropriately according to this recommendation

$$
\begin{array}{ll}
\omega_{l} \ll \min \left\{\omega_{i},\left|\omega_{i}-\omega_{k}\right|,\left|2 \omega_{i}-\omega_{k}\right|\right\}, & \\
\omega_{h}>w_{i}, & \forall i, k \mid i \neq k .
\end{array}
$$




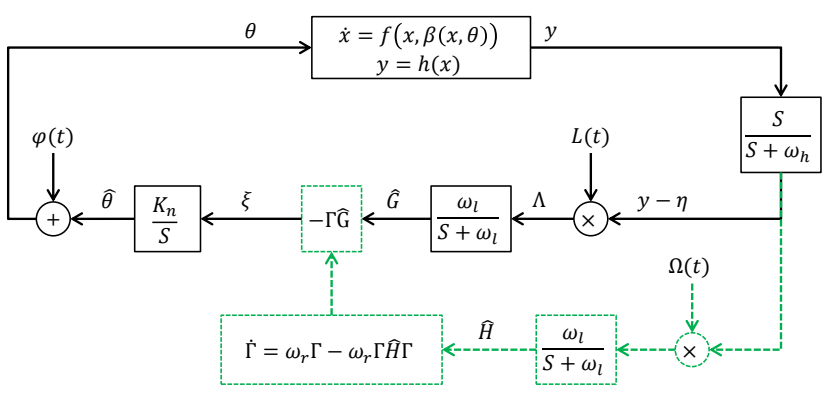

Fig. 6. Block diagram of multivariable Newton-based ES for DCMD.

This design derives an estimate of the gradient vector $\hat{G}$ and the Hessian by adding the perturbation signal $\varphi(t)$ to the estimated optimal input $\hat{\theta}$. The estimate of the Hessian matrix is inverted by the dynamical system given in (24) to avoid difficulties of algebraically inverting $\hat{H}$ when it is close to singular.

$$
\dot{\Gamma}=\omega_{r} \Gamma-\omega_{r} \Gamma \hat{H} \Gamma, \quad \Gamma=\hat{H}^{-1}
$$

The reader is referred to [10] for a detailed convergence proof and design method of the Newton-based ES. To summarize the dynamical system shown in Fig. 6, the following equations are presented.

$$
\begin{aligned}
E \dot{X} & =F(X, \beta(X, \theta)), \\
\dot{\eta} & =-\omega_{h} \eta+\omega_{h} \mathcal{Y}, \\
\dot{\hat{G}} & =-\omega_{l} \hat{G}+\omega_{l} \Lambda, \\
\dot{\hat{\theta}} & =K_{n} \xi, \\
\dot{\hat{H}} & =-\omega_{l} \hat{H}+\omega_{l} \Omega(t)(\mathcal{Y}-\eta), \\
\dot{\Gamma} & =\omega_{r} \Gamma-\omega_{r} \Gamma \hat{H} \Gamma,
\end{aligned}
$$

where

$$
\begin{aligned}
& {\left[\begin{array}{l}
\xi_{1} \\
\xi_{2}
\end{array}\right]=-\left[\begin{array}{ll}
\Gamma_{11} & \Gamma_{12} \\
\Gamma_{21} & \Gamma_{22}
\end{array}\right]\left[\begin{array}{l}
\hat{G}_{1} \\
\hat{G}_{2}
\end{array}\right],} \\
& {\left[\begin{array}{l}
\Lambda_{1} \\
\Lambda_{2}
\end{array}\right]=\left[\begin{array}{l}
L_{1}(t) \\
L_{2}(t)
\end{array}\right](\mathcal{Y}-\eta),}
\end{aligned}
$$

\section{RESUlts AND Discussion}

Simulations were carried out in order to demonstrate the effectiveness of the proposed controller to optimize the solar powered DCMD water desalination process. For this purpose, a realistic membrane parameters were used, as listed in Table I. The design parameters for the Newton-based ES controller are: $c_{1}=c_{2}=0.15, \omega_{1}=3 \mathrm{rad} / \mathrm{s}, \omega_{2}=2 \mathrm{rad} / \mathrm{s}, \omega_{l}=0.1$ $\mathrm{rad} / \mathrm{s}, \omega_{h}=4 \mathrm{rad} / \mathrm{s}, K_{n}=\operatorname{diag}([0.05,0.05]), \omega_{r}=0.0008$.

The feed inlet temperature was designed to reflect a realistic but scaled down in time behavior of a solar powered DCMD setup on a regular day. The temperature profile is depicted in Fig. 7 shown as the dashed line. The permeate inlet temperature is kept constant through out the simulation

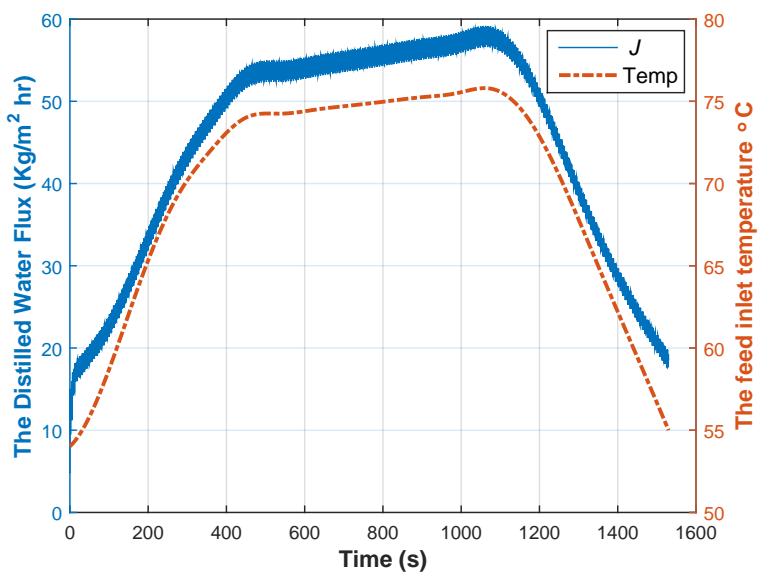

Fig. 7. The distilled water flux $(J)$ is shown on the left axes in solid. The dashed line shows the feed inlet temperature profile.

TABLE I

DCMD MOdule PARAMETERS

\begin{tabular}{l|c}
\hline Property & Value \\
\hline Membrane material & PTFE \\
Pore size & $0.27 \mu \mathrm{m}$ \\
Total thickness & $0.170 \mathrm{~mm}$ \\
Porosity & $77 \%$ \\
Active area & $0.06 \mathrm{~m}^{2}$ \\
Module length & $0.4 \mathrm{~m}$ \\
Module width & $0.15 \mathrm{~m}$
\end{tabular}

at $20^{\circ} \mathrm{C}$. As the feed inlet temperature increase, the distilled water flux is optimized taking into account the feed and permeate inlet mass flow rates, as shown in Fig. 7.

The ES dynamics takes about 50 seconds to converge to the optimal estimated input $\hat{\theta}$ as shown in Fig. 8. After that, the controller tracks the peak of the objective function as it varies with the feed inlet temperature. As the temperature drops, the controller adjusts the inputs immediately.

To demonstrate the effectiveness of the proposed Newtonbased ES, the phase portrait of $\hat{\theta}_{1}$ versus. $\hat{\theta}_{2}$ between time 450 to 500 seconds is shown in Fig. 9. This time window was chosen to be before the feed inlet temperature stagnates at $\approx 75^{\circ} \mathrm{C}$, and thus the Hessian is almost constant approaching the end of the window. It is clear that the input goes straight to the optimum value rather than on a gradient descent like curve.

\section{CONCLUSIONS}

In this paper the optimization of the solar powered DCMD water desalination process was presented. The optimization problem was motivated in order to maximize the distilled water flux while minimizing the feed and permeate inlet mass flow rates, which are highly related to the energy being used to pump water. The multi variable Newton-based ES controller was proposed for real time optimization. A dynamic model of the process was used to carry out the simulation. The results shows that the controller was able to track the peak of the objective function as it varies depending on the feed inlet temperature. These results should be applicable for experimental validations. 

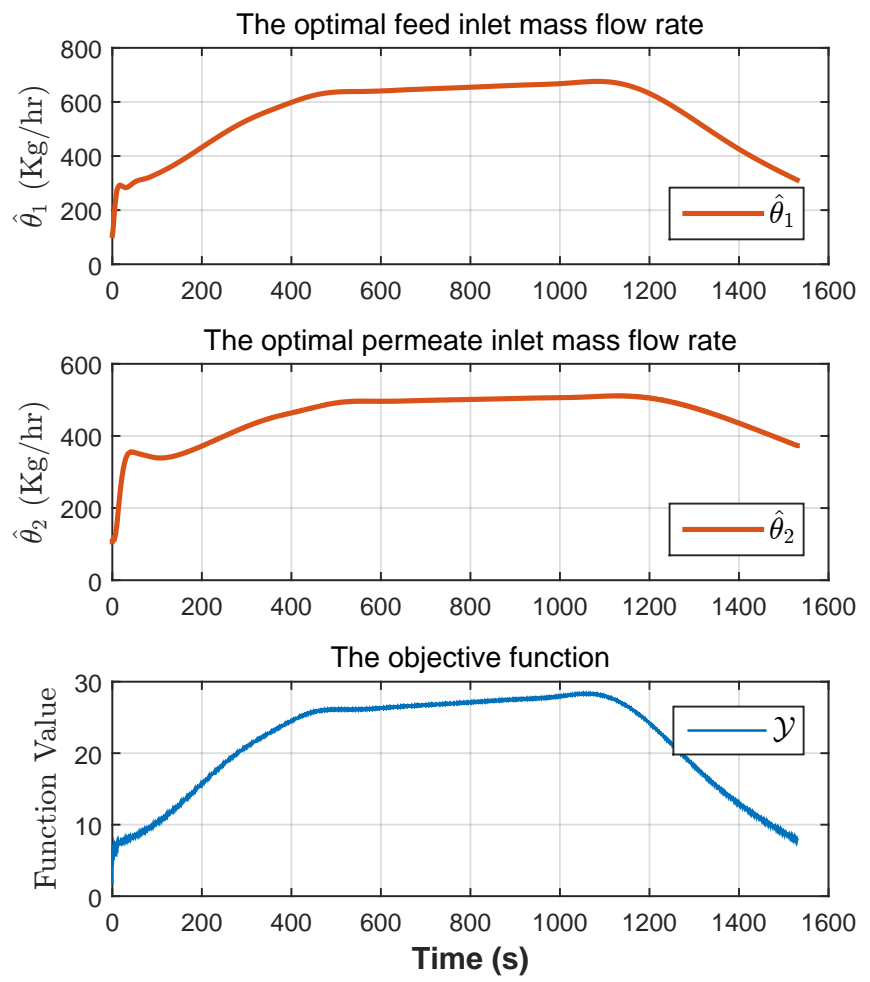

Fig. 8. The estimated optimal inlet mass flow rates for the feed and permeate sides along with objective function value. The quickly Newtonbased ES initializes and then tracks the peak with smooth transitions

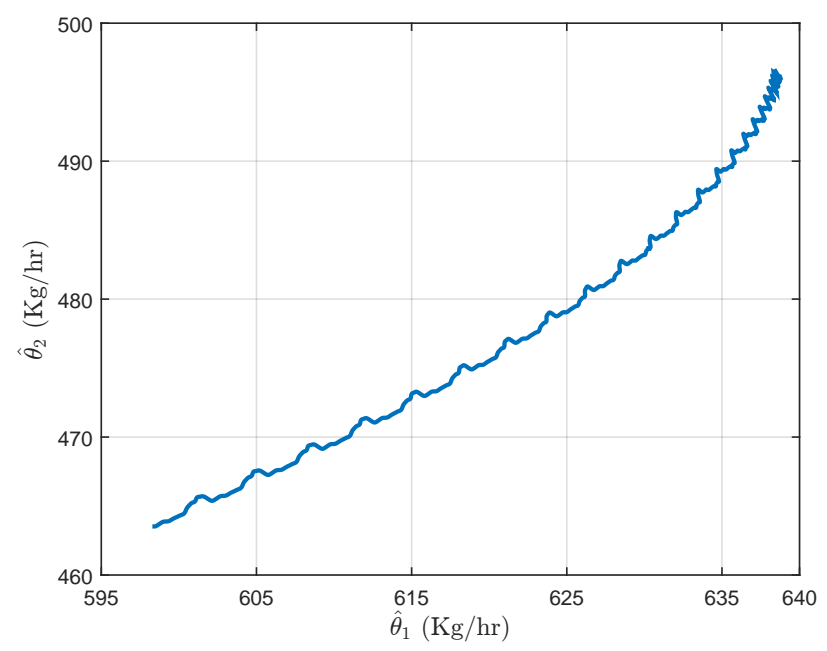

Fig. 9. The phase portrait of $\hat{\theta}_{1}$ versus $\hat{\theta}_{2}$ for the time window between 450 to 500 seconds.

\section{ACKNOWLEDGMENT}

Research reported in this publication was supported by King Abdullah University of Science and Technology (KAUST).

\section{REFERENCES}

[1] J. B. Glvez, L. Garca-Rodrguez, and I. Martn-Mateos, "Seawater desalination by an innovative solar-powered membrane distillation system: the medesol project," Desalination, vol. 246, no. 13, pp. 567 $-576,2009$.

[2] A. Alkhudhiri, N. Darwish, and N. Hilal, "Membrane distillation: A comprehensive review," Desalination, vol. 287, no. 0, pp. 2 - 18, 2012.

[3] H. Chang, G.-B. Wang, Y.-H. Chen, C.-C. Li, and C.-L. Chang, "Modeling and optimization of a solar driven membrane distillation desalination system," Renewable Energy, vol. 35, no. 12, pp. 2714 2722, 2010.

[4] R. Porrazzo, A. Cipollina, M. Galluzzo, and G. Micale, "A neural network-based optimizing control system for a seawater-desalination solar-powered membrane distillation unit," Computers and Chemical Engineering, vol. 54, no. 0, pp. 79 - 96, 2013.

[5] C. E. Garca, D. M. Prett, and M. Morari, "Model predictive control: Theory and practicea survey," Automatica, vol. 25, no. 3, pp. 335 348, 1989.

[6] D. Mayne, J. Rawlings, C. Rao, and P. Scokaert, "Constrained model predictive control: Stability and optimality," Automatica, vol. 36, no. 6, pp. $789-814,2000$.

[7] M. Krstic and H.-H. Wang, "Stability of extremum seeking feedback for general nonlinear dynamic systems," Automatica, vol. 36, no. 4, pp. $595-601,2000$.

[8] N. J. Killingsworth, S. M. Aceves, D. L. Flowers, F. Espinosa-Loza, and M. Krstic, "Hcci engine combustion-timing control: Optimizing gains and fuel consumption via extremum seeking," Control Systems Technology, IEEE Transactions on, vol. 17, no. 6, pp. 1350-1361, Nov 2009.

[9] S. Brunton, C. Rowley, S. Kulkarni, and C. Clarkson, "Maximum power point tracking for photovoltaic optimization using ripple-based extremum seeking control," Power Electronics, IEEE Transactions on, vol. 25 , no. 10 , pp. 2531-2540, Oct 2010 .

[10] A. Ghaffari, M. Krstic, and D. Nesic, "Multivariable newton-based extremum seeking," Automatica, vol. 48, no. 8, pp. 1759 - 1767, 2012.

[11] A. Ghaffari, M. Krstic, and S. Seshagiri, "Power optimization for photovoltaic microconverters using multivariable newton-based extremum seeking," Control Systems Technology, IEEE Transactions on, vol. 22, no. 6, pp. 2141-2149, Nov 2014.

[12] R. Schofield, A. Fane, and C. Fell, "Heat and mass transfer in membrane distillation," Journal of Membrane Science, vol. 33, no. 3, pp. 299 - 313, 1987.

[13] K. W. Lawson and D. R. Lloyd, "Membrane distillation," Journal of Membrane Science, vol. 124, no. 1, pp. 1 - 25, 1997.

[14] A. Karam and T. Laleg-Kirati, "Electrical thermal networks for direct contact membrane distillation modeling," in Control Applications (CCA), 2014 IEEE Conference on, Oct 2014, pp. 1563-1569.

[15] A. M. Karam and T. M. Laleg-kirati, "Electrical thermal network for direct contact membrane distillation modeling and analysis," 2015. [Online]. Available: http://hdl.handle.net/10754/344181 